\section{OC-090 MEASURING THE QUALITY OF SCREENING COLONOSCOPY: MOVING ON FROM ADENOMA DETECTION RATE}

doi:10.1136/gut.2011.239301.90

T J W Lee, ${ }^{1 *}$ C Nickerson, ${ }^{2}$ C J Rees, ${ }^{3}$ on behalf of Northern Region Endoscopy Group (NREG), M D Rutter ${ }^{4}$ on behalf of Northern Region Endoscopy Group (NREG) 'Institute of Health and Society, Newcastle University, Newcastle upon Tyne, UK; ${ }^{2}$ NHS Cancer Screening Programmes, Sheffield, UK; ${ }^{3}$ South of Tyne Bowel Cancer Screening Centre, South Tyneside General Hospital, South Tyneside; ${ }^{4}$ Tees Bowel Cancer Screening Centre, University Hospital of North Tees, Stockton on Tees, UK

Introduction Adenoma detection rate (ADR) is the most widely used metric to assess quality of colonoscopy. Studies have shown ADR to correlate with other measures of colonoscopic performance such as withdrawal time, caecal intubation rate and longer term outcomes such as interval cancer rate.

$\mathrm{ADR}$ is calculated by dividing the number of procedures in which one or more adenomas are detected by the total number of procedures. It has an inherent limitation in that it does not measure the total number of adenomas detected. The ethos of screening colonoscopy, in addition to detecting cancer, is to detect and remove all adenomas.

This abstract examines other measures of adenoma detection in the context of the NHS Bowel Cancer Screening Programme (BCSP).

Methods Data from all screening colonoscopies in the BCSP performed between August 2006 and August 2009 were analysed. The following measures of adenoma detection were calculated for each colonoscopist who had performed $\geq 50$ procedures: ADR, MAP (mean adenomas per patient-total number of adenomas detected/ number of procedures), MAP+ (mean adenomas per positive procedure-total number of adenomas detected/ number of procedures in which one or more adenoma were detected). Correlation was assessed using Pearson's correlation coefficient.

This abstract examines other measures of adenoma detection in the context of the NHS BCSP.

Results 31088 procedures performed by 148 colonoscopists (mean 210 procedures per colonoscopist) were analysed. The mean, range, SD and correlation with ADR of each of the measures of adenoma detection are shown in table 1.

$46.5 \%$ of patients have one or more adenomas, of these $53 \%$ have only one adenoma. For this reason, MAP and ADR correlate closely. However, MAP displays much greater variation than ADR. MAP+ demonstrated less variation and correlates less well with ADR.

Conclusion MAP reflects the aim of screening colonoscopy to detect and remove all adenomas. MAP may be a more sensitive measure of colonoscopic performance but prospective studies are required to confirm this.

MAP+ is a less useful measure of adenoma detection in isolation. It is largely dependent on the ADR but may still be high despite a low ADR. It may be useful as an adjunct to ADR or MAP in colonoscopists with evidence of high colonoscopic performance (ADR greater than the mean of the population). In this group, MAP+ identifies a group of colonoscopists attaining very high adenoma detection.

Competing interests None.

Keywords adenoma, colonoscopy, colorectal cancer, screening.

Table 1 OC-090 ADR, MAP and MAP+, summary data

\begin{tabular}{lccccc}
\hline & Mean & Range & SD & $\begin{array}{c}\text { Variation between } \\
\text { highest and lowest } \\
\text { colonoscopists }\end{array}$ & $\begin{array}{c}\text { Correlation with } \\
\text { ADR }(\mathbf{r})\end{array}$ \\
\hline ADR & $46.5 \%$ & $21.9-59.8 \%$ & $7 \%$ & Threefold & N/A \\
MAP & 0.91 & $0.31-1.86$ & 0.25 & Sixfold & $0.85(\mathrm{p}<0.001)$ \\
MAP+ & 1.94 & $1.3-3.1$ & 0.35 & 1.3 -fold & $0.54(\mathrm{p}<0.001)$ \\
\hline
\end{tabular}

